\title{
A combined therapeutic approach to treating a challenging case of Dens Invaginatus
}

SADJ March 2020, Vol. 75 No. 2 p81 - p86

CH Jonker ${ }^{1}$, P van der Vyver ${ }^{2}$, S Arnold ${ }^{3}$, NH Wood $^{4}$

\section{ABSTRACT}

\section{Background and objectives}

Dens Invaginatus is a rare dental anomaly. The case presentation illustrates a combined endodontic and surgical approach of a maxillary lateral incisor that presented with a Type III Dens Invaginatus.

\section{Methods}

The complex root canal system was prepared during phase 1 after proper disinfection; the canals were obturated with a combination of techniques.

An appointment was scheduled for four weeks later for the surgical phase. During this visit, a full muco-periostal flap was raised, the failed retrograde restoration was removed and MTA was placed.

\section{Author affiliations:}

1. Casper H Jonker: BChD, Dip Odont, MSc (Pret), Module of Endodontics, Department of Operative Dentistry, School of Oral Health Sciences, Sefako Makgatho Health Sciences University, Gauteng, South Africa.

ORCID Number: 0000-0002-9110-5208

2. Peet J van der Vyver: BChD, Dip Odont, MSc, PhD (Pret), Department of Odontology, School of Dentistry, University of Pretoria, Pretoria, South Africa and Private Practice, Sandton, South Africa.

ORCID Number: 0000-0003-1951-6042

3. Samantha Arnold: BChD, PG Dip Dent (Pret), Department of Odontology, School of Dentistry, University of Pretoria, Pretoria, South Africa.

ORCID Number: 0000-0002-7518-9560

4. Neil H Wood: BChD, Dip Dont, MDent (OMP), Department of Periodontology and Oral Medicine, School of Oral Health Sciences, Sefako Makgatho Health Sciences University, Gauteng, South Africa.

ORCID Number: 0000-0001-8950-7999

Corresponding author: Casper $\mathrm{H}$ Jonker

School of Oral Health Sciences, Sefako Makgatho Health Sciences

University, Gauteng, South Africa.

Email: casper.jonker@smu.ac.za

Author contributions:

1. Casper $\mathrm{H}$ Jonker: Introduction (25\%), report on treated case and procedures $(80 \%)$, discussion and literature review $(25 \%)$, conclusion (25\%), changes and formulation of drafts (30\%).

2. Peet $\mathbf{J}$ van der Vyver: Introduction (25\%), discussion and literature review $(25 \%)$, conclusion $(25 \%)$, changes and formulation of drafts (30\%).

3. Samantha Arnold: Introduction (25\%), discussion and literature review $(25 \%)$, conclusion $(25 \%)$, changes and formulation of drafts $(20 \%)$.

4. Neil H Wood: Introduction (25\%), report on treated case and procedures (20\%), discussion and literature review (25\%), conclusion $(25 \%)$, changes and formulation of drafts $(20 \%)$.

\author{
ACRONYMS \\ DI: Dens Invaginatus \\ DFDBA: Demineralized Freeze-Dried Bone Allograft \\ SLA: $\quad$ Straight Line Access
}

\section{Results}

The patient was seen for follow-up visits after the procedure and satisfactory healing was observed.

\section{Conclusion}

The case report illustrates a novel approach to treating a unique case of dens in dente.

\section{INTRODUCTION}

Dens Invaginatus (DI) is a rare dental anomaly in which invagination of the enamel organ into the dental papilla occurs before tooth calcification. The invagination may originate in the crown region of the affected tooth, often extending into the root before calcification of tooth structure occurs.

Various other terms can be found for this phenomenon: "dens in dente", "dilated composite odontome", "gestant odontoma", "dentinoid in dente" or "telescopic tooth". ${ }^{2}$ Teeth affected by this anomaly may show a great variety of sizes, shapes and forms. ${ }^{3}$ Salter gave a very accurate description of this anomaly in a very early statement in 1855, in which it was named "a tooth within a tooth", ${ }^{4}$ and was also the first to describe DI in a human tooth. ${ }^{3}$

Once the diagnosis of a DI is confirmed, treatment can pose a formidable challenge to the treating clinician and modern clinical techniques and equipment may be required for successful management. Radical approaches in treatment, including endodontics, are needed, and often the affected tooth has to be extracted. ${ }^{3,4,5}$ These teeth may pose challenges concerning aesthetics and occlusion, a higher risk of caries, pulp necrosis and finally tooth impaction. ${ }^{5}$

The following case presentation demonstrates an endodontic and surgical approach to treating a challenging case of DI. 


\section{CASE REPORT}

\section{Conventional root canal treatment}

A 29-year-old male patient with an uncomplicated medical history was referred to the Department of Operative Dentistry at the Oral Health Centre, Sefako Makgatho Health Sciences University for endodontic treatment on his maxillary right second incisor. According to the patient, a root canal treatment had been attempted during a previous visit to the hospital.

On clinical examination a discoloured maxillary right lateral incisor with a slight class I mobility was seen. A periapical radiograph of the area revealed a complex root canal configuration with a large retrograde amalgam restoration at the apex of the root (Figure 1).

Small fragments of the retrograde amalgam were scattered around the root apex and surrounding tissues.

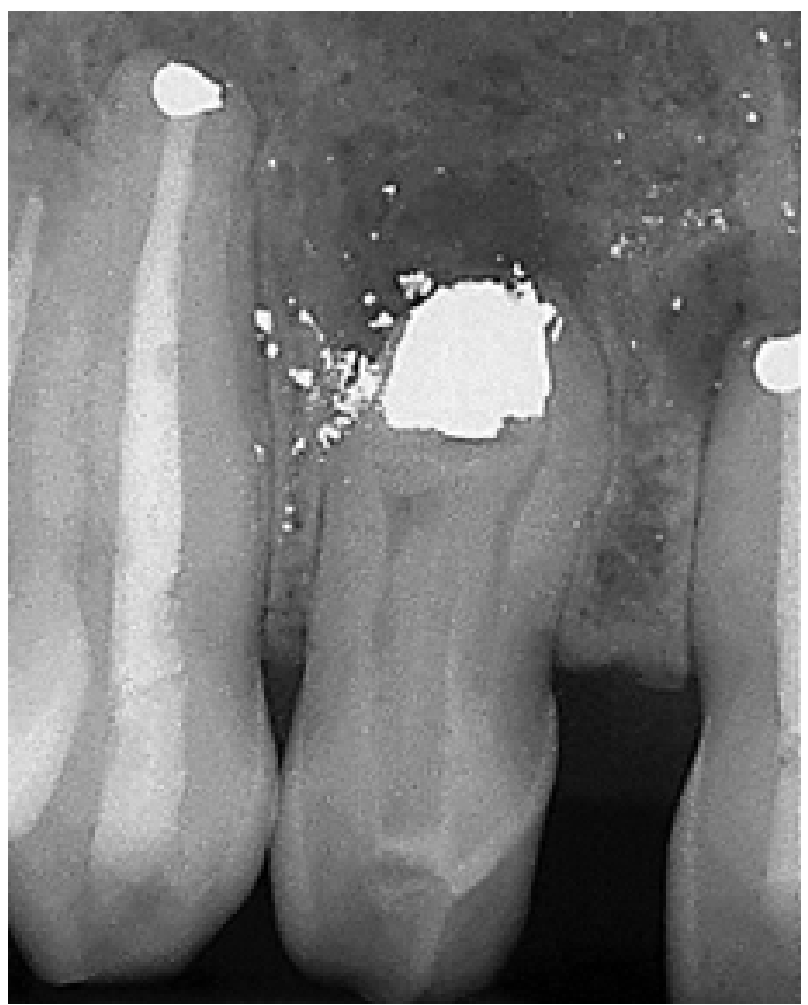

Figure 1. Pre-operative radiograph revealing the complex anatomy of the root canal system and failed treatment with a large retrograde restoration and restorative particles visible in the apical area of the right maxillary lateral incisor.

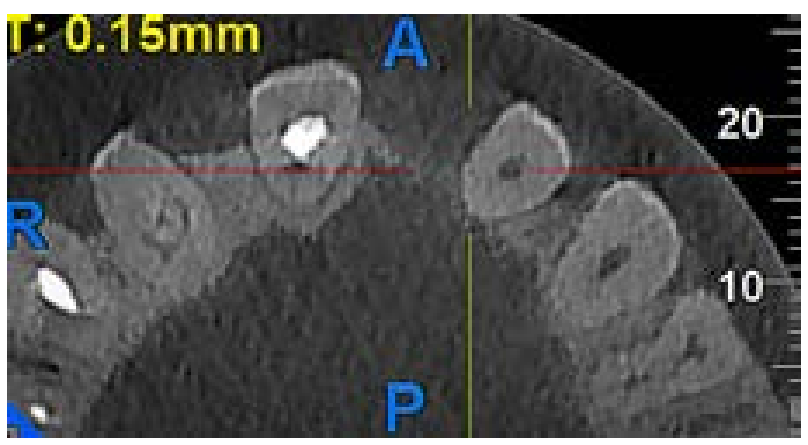

Figure 2. Axial slice of CBCT scan confirming the central root with root canal enclosed by a $\mathrm{C}$-shaped root canal space of the right maxillary lateral incisor.
There was also an apical radiolucency around the apex of the root, and evidence of previous endodontic treatment was visible. The right central incisor and canine had been treated previously by means of conventional endodontic treatment with retrograde amalgam restorations. A periapical radiolucency was visible around the apex of the maxillary right central incisor.

A DI Type III was diagnosed and the decision was made to take a cone beam computed tomography (CBCT) scan. The CBCT images confirmed the complexity of the canal systems, in which the central root canal was enclosed by another C-shaped pulp chamber (Figure 2).

After discussion with the patient and obtaining his consent for treatment, the tooth was anaesthetised, a rubber dam placed, the temporary restoration removed and straight line access (SLA) achieved. The main central root canal system was identified under high magnification using the Dental Operating Microscope (DOM) (Carl Zeiss, Oberkochen, Germany). The complex root canal configuration was scouted with manual K-files (Dentsply-Sirona, Ballaigues, Switzerland) (Figure 3) but no patency was achieved due to the retrograde restoration.

Working length was determined by using a size 0.8 $\mathrm{K}$-file and confirmed radiographically. In the central root canal, glide path preparation was initiated with a size $10 \mathrm{~K}$-file and the glide path was expanded with the Proglider (Dentsply Sirona), a single rotating glide path file.

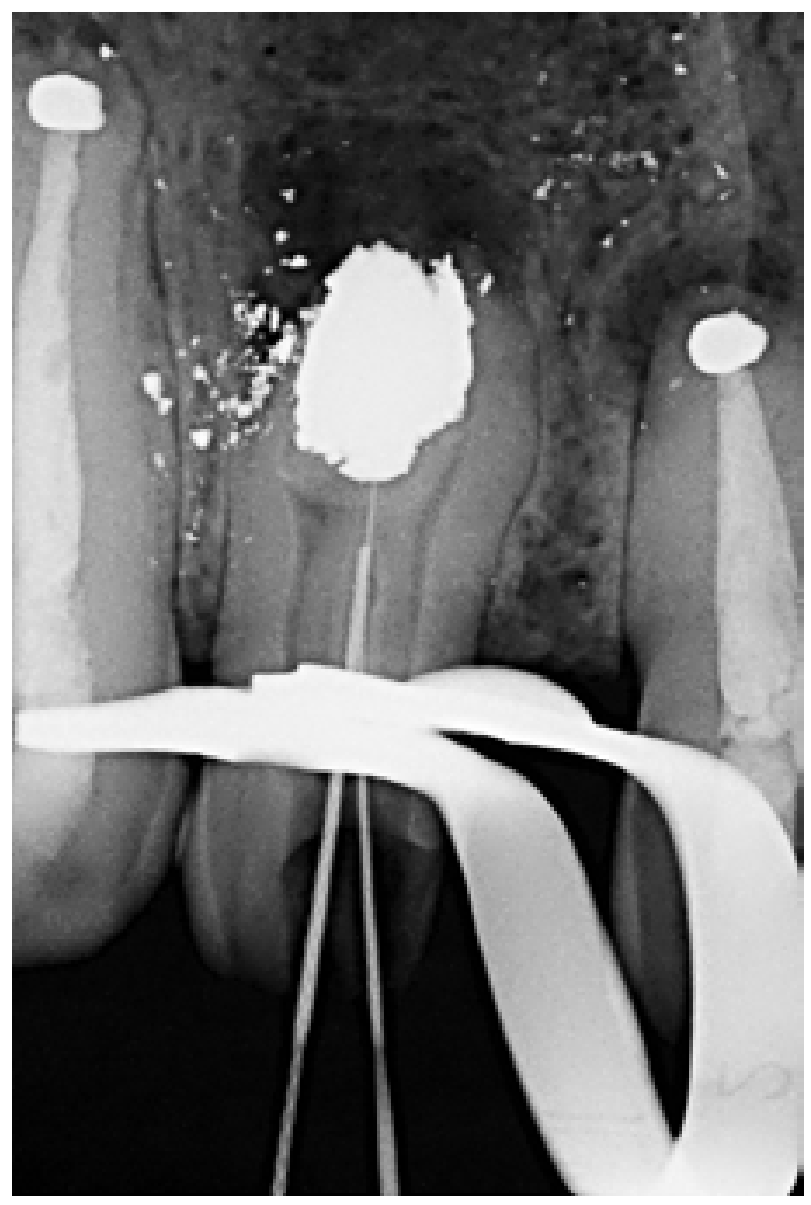

Figure 3. Scouting of the root canal configuration using K-files. Instruments were not able to achieve apical patency. 
Root canal preparation was done with a WaveOne Gold Primary endodontic file (Dentsply Sirona). RC-Prep (Premier Dental, Plymouth Meeting, Pennsylvania) was used as a lubricant, and for irrigation between the instruments $3.5 \%$ sodium hypochlorite solution was activated with the EndoActivator (Dentsply Sirona).

Under high magnification, the C-shaped root canal space was investigated for additional root canal orifices or hidden anatomy. An ultrasonic tip, Start-X number 3 (Dentsply Sirona) was used to open root canal space and gain improved access to the C-shaped canal configuration. The WaveOne Gold Primary file was used in a brushing motion on the outstroke in all directions in an effort to shape the C-shaped root canal space. Final root canal irrigation was done with $3.5 \%$ sodium hypochlorite solution that was again activated with the EndoActivator (Dentsply Sirona) to improve disinfection in the C-shaped root canal configuration.

The root canal systems were dried with paper points and the canals obturated with WaveOne Gold Primary gutta-percha cones (Dentsply Sirona) and BioRoot RCS endodontic sealer (Septodont, Saint-Maur-desFossés, France) using the Calamus Dual Obturation Unit (Dentsply Sirona) (Figure 4). The tooth was restored and an appointment was scheduled for the surgical exploration of the periapical lesion.

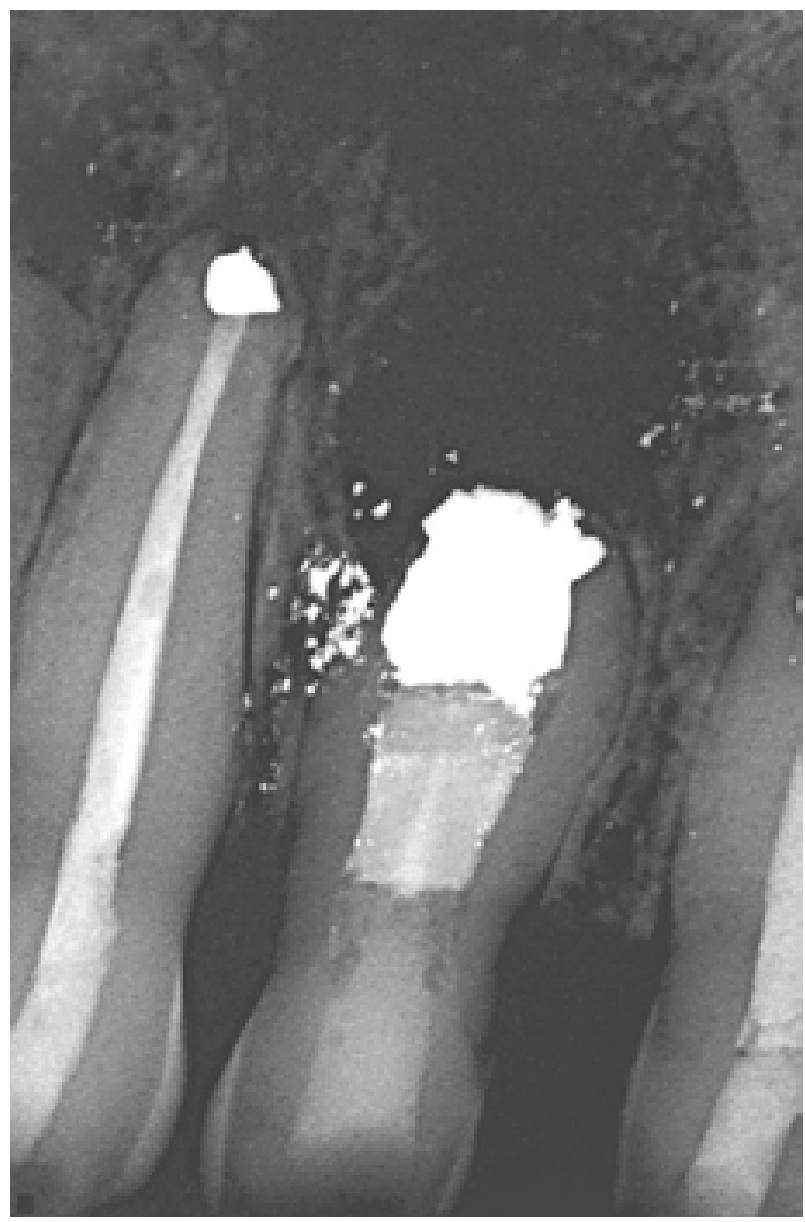

Figure 4. Post-obturation radiograph and restoration completing phase one of the treatment. The obturation was completed on the main centra root canal and $\mathrm{C}$-shaped root canal space using a combination of obturation techniques.

\section{Surgical treatment}

The patient returned four weeks later for the surgical phase of the treatment. The surgical site was anaesthetised and a full muco-periostal flap was raised. Bone was removed at a level corresponding to the apical region of the lateral incisor.

The apical region was explored and it was confirmed that a previously placed amalgam retrograde restoration was firmly attached to the apex of the root.

The retrograde amalgam restoration was removed using an ultrasonic tip (Start-X number 3, Dentsply Sirona) and a long diamond burr (Komet Dental, Brasseler, Germany) with constant water cooling.

The remaining apex of the root was observed under magnification to identify all the portals of exit. The decision was made to leave any amalgam scattering in situ to avoid possible further damage to apical tissues and root structures. No other portals of exit were identified. Mineral Trioxide Aggregate (MTA) (Dentsply Sirona) was mixed according to the manufacturer's instructions and placed as retrograde restoration.

The apical regions of the canine and central incisor were assessed intra-operatively. No pathology was observed at the canine apex, but granulation tissue was removed around the apex of the central incisor. The integrity of the existing retrograde restorations were intact and, as no signs of leakage were observed, these were not replaced.

Bio-resorbable demineralised freeze-dried bone allograft (DFDBA) (Osseograft TM, Advanced Biotech Products, Chennai, India) was used to fill the bone defects and primary closure of the surgical site was achieved. A follow-up appointment was scheduled for one week later and post-operative instructions were provided.

\section{Follow-up visits}

The patient was seen after 12 weeks (Figure 5) for a follow-up visit, reporting no discomfort. The surgical site was inspected and uneventful healing was observed. No mobility of the tooth was detected.

At a 13 month follow-up visit a periapical radiograph revealed satisfactory healing of the periapical pathology around the roots of the central and lateral incisor teeth (Figure 6).

\section{Discussion and literature review}

Back in 1957, Oehlers described three types of DI (Figure 7): Type 1: minor invagination limited to the crown, not extending beyond the cemento-enamel junction; Type II: apical extending invagination not limited to the coronal region, but extending beyond the cementoenamel junction, forming a blind sac inside the root, which may or may not communicate with the pulp; Type III: severe apical extension of the invagination into the root and exiting into the periodontium. ${ }^{6}$ 


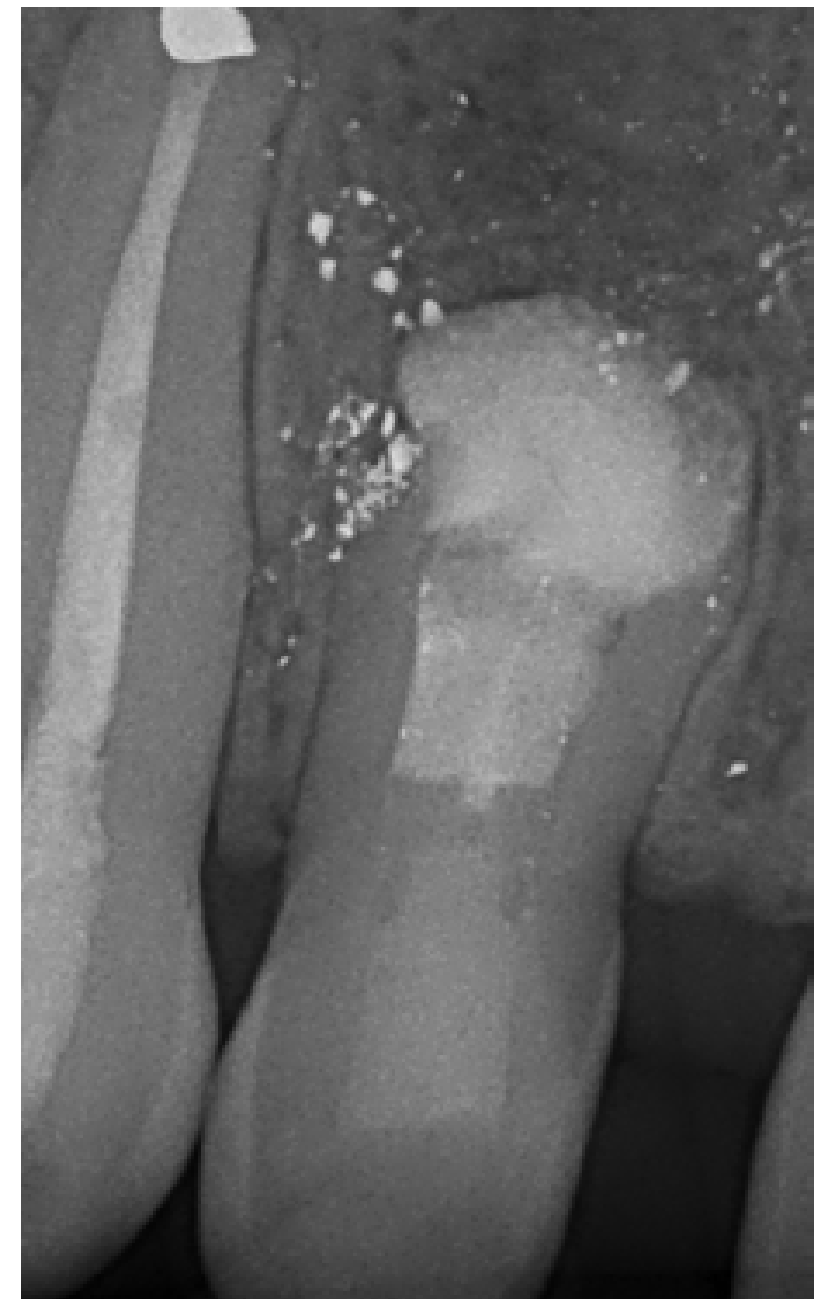

Figure 5. Three-month follow-up periapical radiograph indicating early signs of healing of the apical radiolucency and improved integrity of the apical bone network.

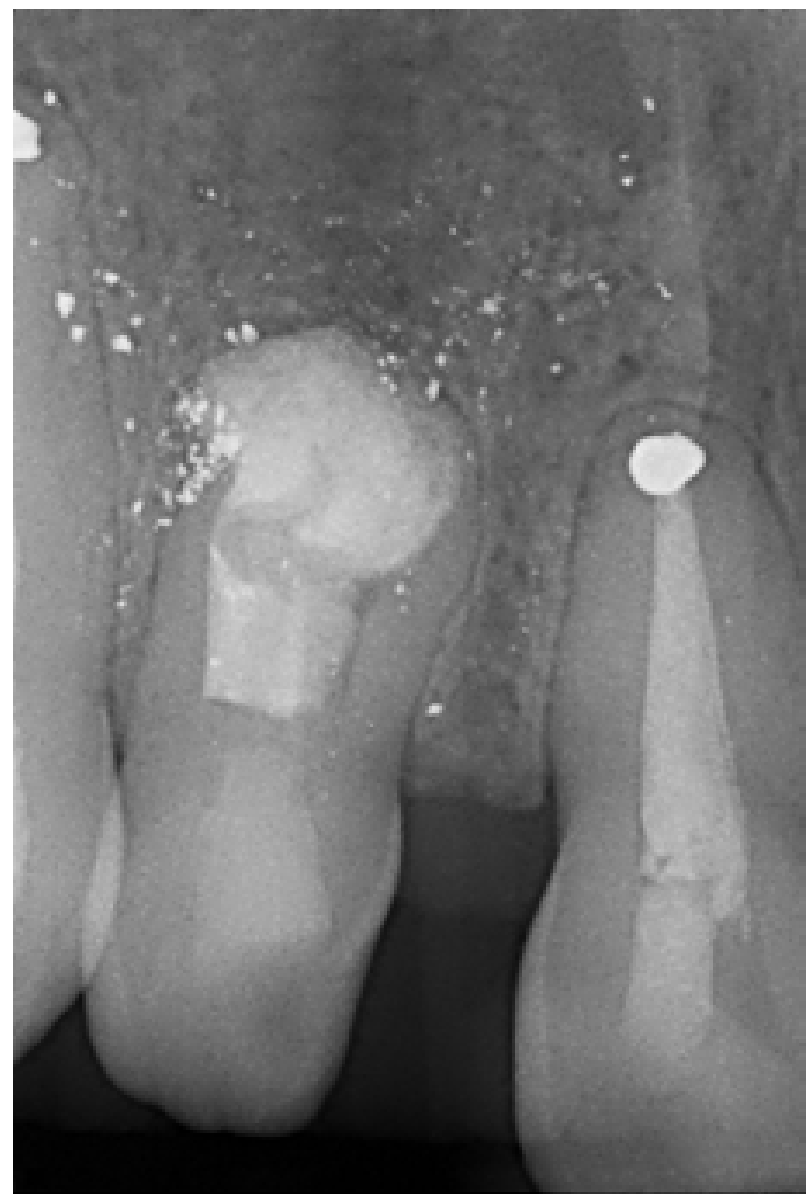

Figure 6. Thirteen-month follow-up images after the surgical phase of treatment: conventional radiograph indicates almost complete healing of the surgical site and apical area.

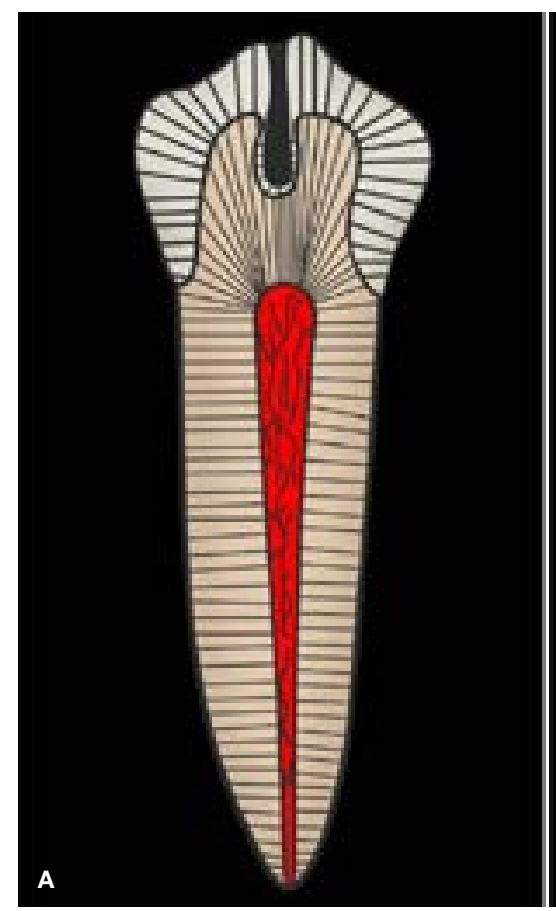

Figure 7. Schematic representation of the different types of dens invaginatus:

(A) Type I: Minor invagination limited to the crown, not extending beyond the cemento-enamel junction;

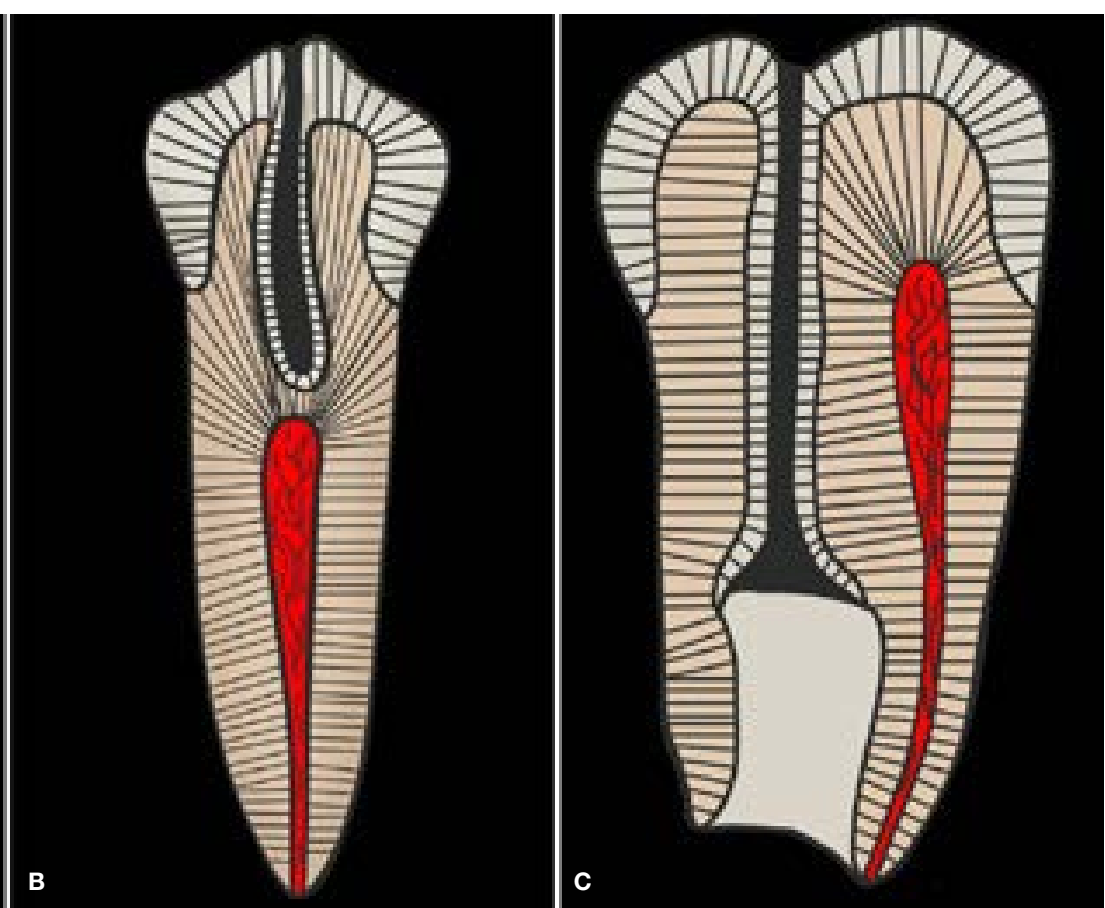

(B) Type II: Apical extending invagination not limited to the coronal region, but extending beyond the cemento-enamel junction, forming a blind sac inside the root, which may or may not communicate with the pulp; (C) Type III: Severe apical extension of the invagination into the root and exiting into the periodontium. ${ }^{6}$ 
Type III can further be divided into Types III a and b. With Type III a, the invagination extends into the root and exits laterally into the periodontal space; pulpal involvement is unlikely. ${ }^{7,8}$ Type III b shows an invagination extending into the root and exiting at the apical foramen into the periodontal space. ${ }^{7,8}$ In rare cases of Type III b, the invagination can be outlined by cementum. ${ }^{8}$ According to the literature, Oehlers' radiographic classification is still used more frequently today than any other. ${ }^{9}$

There is no consensus on the aetiology of this phenomenon. Proposals as to the formation of this "tooth within a tooth" include lateral fusion of two tooth germs, the dental arch imposing constriction within the enamel organ, increased external pressure on the developing tooth germ, restriction of focal growth, individual stimulation of various areas within the tooth bud, crown invagination before calcification of the tooth, infection during the time of tooth development and finally, possible genetic factors. ${ }^{10,11,12}$

Clinical signs alerting the clinician to the possible presence of the $\mathrm{DI}$ are a deviation in normal crown morphology, including dilated, peg-shaped or barrelshaped crowns. $^{7}$ An enlarged palatal cingula, notching of the incisal edges with palatal grooving, talon cusps and a deep foramen caecum will also raise suspicion. ${ }^{7,10}$ Methylene blue dye can be used to distinguish the normal fissures from the foramen caecum. ${ }^{8}$ Unfortunately, affected teeth can also present with no clinical signs.

The literature records that maxillary lateral incisors are more susceptible to coronal invaginations ${ }^{13}$ than any other tooth in the human dentition. Studies have also confirmed a prevalence in the region of $0.04 \%$ to $10 \%$ with a female: male ratio of $3: 1.3,14$ Invaginations in posterior teeth are not common, and Hamasha and Al-Omari $(2004)^{15}$ found only $6.5 \%$ of affected teeth were in the posterior region.

Furthermore, the maxilla is more often affected and DI is also more commonly found in the Caucasian and Asian races. The phenomenon can also be associated with various syndromes: Ekman-Westborg-Julin syndrome, Williams syndrome and Nance Huran syndrome. ${ }^{16}$

Clinicians must be encouraged to spend more time on a thorough examination of teeth, especially where deep pits are observed in the region of the foramen caecum. Hypo-mineralisation of the enamel in the region of the dens may be detected, which may make the spread of bacteria easier and faster. ${ }^{8}$ Subsurface caries may develop in the DI because of the tortuous lingual anatomy of the anterior teeth, initially being confined to the internal surface.

The caries could later progress to the external surface or to the pulp through pathways between the dens and the pulp. ${ }^{8}$ With early detection, the need for complex and difficult endodontic treatments at a later stage will be averted. ${ }^{17}$ The investigating clinician is also encouraged to thoroughly examine the contra-lateral tooth, as $43 \%$ of patients can present with the same anomaly in this tooth. ${ }^{18}$ Patients with a DI can seek dental treatment for acute pain and inflammation, but in general the diagnosis of $\mathrm{DI}$ is confirmed during routine examinations, panoramic $x$ rays and CBCT scans. Once the suspicion is raised, a periapical radiograph will confirm the finding. ${ }^{11}$

The radiological appearance is determined by the severity of the individual case. One case may present with a single deep, narrow pit whilst another may present with an in-folding loop very close to the main root canal. Radiopaque enamel lining is mostly present ${ }^{7,8}$ and the pulp can display a very complex image of anatomy, difficult to follow and interpret. ${ }^{19}$ Radiographs can only detect relevant information in the coronal plane of anterior teeth and in the sagittal plane of posterior teeth. ${ }^{17}$

Today, the use of CBCT scans in conjunction with traditional radiographs is highly advisable. CBCT provides a non-invasive 3D reconstructed view of external and internal morphological challenges of the DI tooth. ${ }^{17,20}$ Because of the relatively higher dose of radiation involved, the current recommendation is that CBCT scanning should be reserved for selected cases where conventional radiographs fail to provide conclusive information on the complex endodontic configuration. ${ }^{17}$

Histologically the enamel of the DI appears hypo-mineralised and may have an altered chemical composition when compared with the external enamel. The dentine of the DI may be regularly or irregularly hypo-mineralised. It may also contain vital connective tissue strains and show intercommunications with the pulp. ${ }^{8}$

Minimal invasive procedures are the aim when treating DI. Conservative restoration of the invagination with fissure sealant or flowable composite is the treatment of choice when the coronal caries are shallow, the pulp tests are vital and periapical lesions are absent. ${ }^{7}$ Periodic followups of clinical and radiographic examinations of these cases are of utmost importance.

DI teeth with vital pulps may present with apical periodontitis, therefore pulp tests are essential for proper pulpal diagnosis, irrespective of what the radiograph presents. ${ }^{7}$ The more extensive the invagination, the more invasive the treatment will be; each case requires a different approach.

The present case describes treatment of a non-vital lateral incisor with a DI Type II and associated periapical pathology. Root canal therapy of both the invagination and the main root canal, together with apical surgery, were needed to resolve the periapical pathosis.

Root canal therapy in DI poses challenges and limitations, as the complex root canal anatomy together with the obstructive invagination limit accessibility and make complete debridement quite difficult. ${ }^{21}$

\section{CONCLUSION}

The case report illustrated a novel approach to treating a unique case of dens invaginatus. A conventional endodontic approach was combined with a surgical 
approach and at a 13-month follow-up, the tooth and surrounding apical tissue showed adequate signs of healing.

\section{Declaration}

The authors declare they have no conflict of interest in the publishing of this manuscript. The manuscript was processed by the outgoing managing editor, Prof Bill Evans.

\section{References}

1. Zengin AZ, Sumer AP, Celenk P. Double dens invaginatus: report of three cases. Eur J Dent. 2009; 3: 67-70.

2. Sedano HO, Ocampo-Acosta F, Naranjo-Corona RI, TorresArellano ME. Multiple dens invaginatus, mulberry molar and conical teeth. Case report and genetic considerations. Med Oral Patol Oral Cir Bucal. 2009; 14: E69-72.

3. Hülsmann M. Dens invaginatus: aetiology, classification, prevalence, diagnosis, and treatment considerations. Int Endod J. 1997; 30: 79-90.

4. Silberman A, Cohenca N, Simon JH. Anatomical redesign for the treatment of dens invaginatus type III with open apexes: a literature review and case presentation. J Am Dent Assoc. 2006; 137: $180-5$.

5. De Rossi A, De Carvalho FK, De Queiróz AM, Bezerra da Silva RA, Nelson-Filho P, Bezerra da Silva LA. The treatment of a maxillary lateral incisor with unusual morphology with long-term follow-up. Int Endod J. 2013; 46: 1183-90.

6. Oehlers FAC. Dens invaginatus I: variations of the invagination process and associated anterior crown forms. Oral Surg Oral Med Oral Pathol. 1957; 10: 1204-18.

7. Martins JNR, Da Costa RP, Anderson C, Quaresma SA, Corte-Real LSM, Monroe AD. Endodontic management of dens invaginatus type Illb: case series. Eur J Dent. 2016; 10: 561-5.

8. Sisodia N, Manjunath MK. Double dens in dente: a rare anomaly. S Afr Dent J. 2016; 71: 410 -11.
9. Skrinjaric T, Gorseta K, Skrinjaric I. Lobodontia: genetic entity with specific pattern of dental dysmorphology. Ann Anat. 2016; 203: 100 -7.

10. Mupparapu M, Singer SR. A rare presentation of dens invaginatus in a mandibular lateral incisor occurring concurrently with bilateral maxillary dens invaginatus: case report and review of literature. Aust Dent J. 2004; 49: 90 -3.

11. Suprabha BS. Premolarized double dens in dente in albinism - a case report. J Indian Soc Pedod Prev Dent. 2005; 23 $156-8$.

12. Regezi JA, Sciubba JJ. Oral pathology clinical pathologic correlations. In: Abnormalities of teeth $\left(4^{\text {th }}\right.$ ed.) Saunders, 2003; $369-70$.

13. More $\mathrm{CB}$, Patel HJ. Dens invaginatus: a radiographic analysis 2012; 1:147. doi:10.4172/scientificreports.147.

14. Beena VT, Sivakumar R, Heera R, Rajeev R, Choudhary K, Panda S. Radicular dens invaginatus: report of a rare case. Case Rep Dent. 2012; 87: 1937.

15. Hamasha AA, Al-Omari QD. Prevalence of dens invaginatus in Jordanian adults. Int Endod J. 2004; 37: 307-10.

16. Mupparapu M, Singer SR, Pisano D. Diagnosis and clinical significance of dens invaginatus to practicing dentists. NY State Dent J. 2006; 72: 42-6.

17. Capar ID, Ertas H, Arslan H, Ertas ET. A retrospective comparative study of cone-beam computed tomography versus rendered panoramic images in identifying the presence, types and characteristics of dens invaginatus in a Turkish population. J Endod. 2015; 41: 473-8.

18. Bishop K, Alani A. Dens invaginatus. part 2: clinical, radiographic features and management options. Int Endod J. 2008; 41: 1137-54.

19. Patel S. The use of cone beam computed tomography in the conservative management of dens invaginatus: a case report. Int Endod J. 2010; 43: 707-13.

20. Dinapadu S, Aravelli S, Pasari S, Marukala NR. A rare presentation of bilateral maxillary dens invaginatus diagnosed using cone beam computed tomography. J Clin Imaging Sci. 2013; 3 (Suppl 1): 8.

21. Shadmehr E, Kiaani S, Mahdavian P. Nonsurgical endodontic treatment of a maxillary lateral incisor with dens invaginatus type II: a case report. Dent Res J. 2015; 12: 187-91.

\section{Do the CPD questionnaire on page 104}

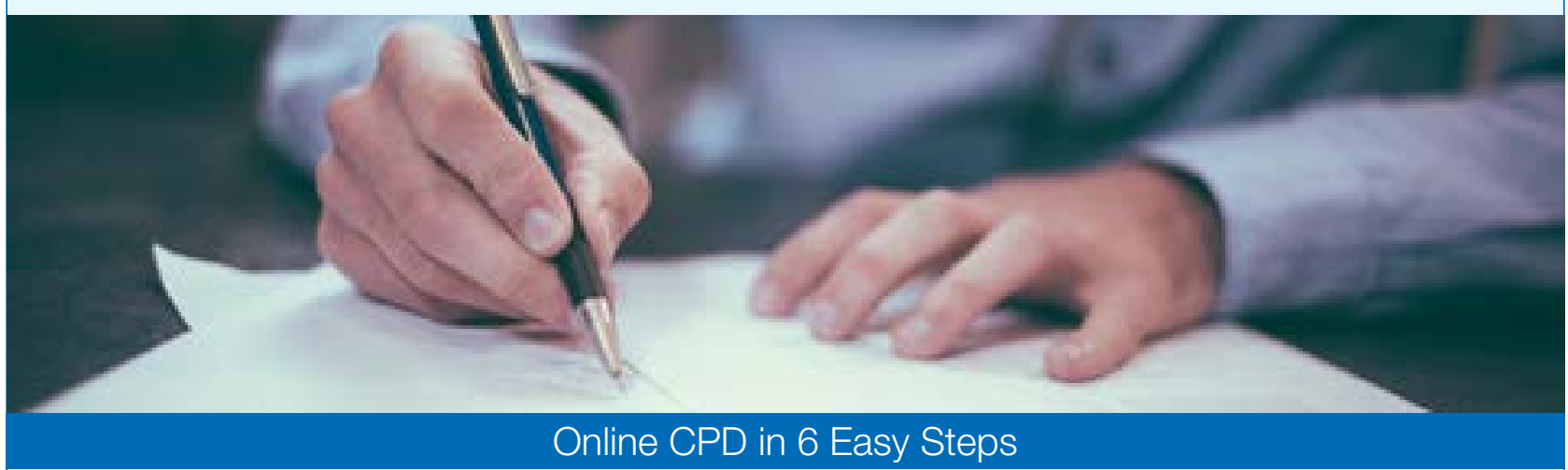

1 Go to the SADA website www.sada.co.za.

2 Log into the 'member only' section with your unique SADA username and password.

3 Select the CPD navigation tab.

4 Select the questionnaire that you wish to complete.

5 Enter your multiple choice answers. Please note that you have two attempts to obtain at least $70 \%$.

6 View and print your CPD certificate. 